\title{
Hybrid Deep Learning for Medication-Related Information Extraction From Clinical Texts in French: MedExt Algorithm Development Study
}

Jordan Jouffroy $^{1,2}$, MD, MSc; Sarah F Feldman ${ }^{1,2}$, MD; Ivan Lerner ${ }^{1,2}$, MD; Bastien Rance ${ }^{2,3}$, PhD; Anita Burgun ${ }^{1,2}$, $\mathrm{MD}, \mathrm{PhD} ;$ Antoine Neuraz ${ }^{1,2}, \mathrm{MD}, \mathrm{PhD}$

\footnotetext{
${ }^{1}$ Department of Biomedical Informatics, Necker-Enfants malades Hospital, Assistance Publique-Hôpitaux de Paris, Paris, France

${ }^{2}$ UMRS 1138 team 22, Institut National de la Santé et de la Recherche Médicale, Université de Paris, Paris, France

${ }^{3}$ Department of Biomedical Informatics, Georges Pompidou European Hospital, Assistance Publique-Hôpitaux de Paris, Paris, France
}

\section{Corresponding Author:}

Antoine Neuraz, MD, PhD

Department of Biomedical Informatics

Necker-Enfants malades Hospital

Assistance Publique-Hôpitaux de Paris

Bâtiment Imagine - Bureau 145

149 rue de Sèvres

Paris,

France

Phone: 33171396585

Email: antoine.neuraz@aphp.fr

\section{Abstract}

Background: Information related to patient medication is crucial for health care; however, up to $80 \%$ of the information resides solely in unstructured text. Manual extraction is difficult and time-consuming, and there is not a lot of research on natural language processing extracting medical information from unstructured text from French corpora.

Objective: We aimed to develop a system to extract medication-related information from clinical text written in French.

Methods: We developed a hybrid system combining an expert rule-based system, contextual word embedding (embedding for language model) trained on clinical notes, and a deep recurrent neural network (bidirectional long short term memory-conditional random field). The task consisted of extracting drug mentions and their related information (eg, dosage, frequency, duration, route, condition). We manually annotated 320 clinical notes from a French clinical data warehouse to train and evaluate the model. We compared the performance of our approach to those of standard approaches: rule-based or machine learning only and classic word embeddings. We evaluated the models using token-level recall, precision, and F-measure.

Results: The overall F-measure was $89.9 \%$ (precision 90.8; recall: 89.2) when combining expert rules and contextualized embeddings, compared to $88.1 \%$ (precision 89.5; recall 87.2) without expert rules or contextualized embeddings. The F-measures for each category were $95.3 \%$ for medication name, $64.4 \%$ for drug class mentions, $95.3 \%$ for dosage, $92.2 \%$ for frequency, $78.8 \%$ for duration, and $62.2 \%$ for condition of the intake.

Conclusions: Associating expert rules, deep contextualized embedding, and deep neural networks improved medication information extraction. Our results revealed a synergy when associating expert knowledge and latent knowledge.

(JMIR Med Inform 2021;9(3):e17934) doi: 10.2196/17934

\section{KEYWORDS}

medication information; natural language processing; electronic health records; deep learning; rule-based system, recurrent neural network; hybrid system 


\section{Introduction}

In 2017, medication consumption in France represented $€ 37.8$ billion (approximately US $\$ 45.5$ billion) in spending and $16 \%$ of the French health budget [1]. Adverse drug reactions are an important public health problem, representing a major cause of mortality ( $0.15 \%$ in France); one-third of admissions caused by adverse drug reactions are preventable, associated with a poorly reported drug history or rare adverse events $[2,3]$.

Furthermore, electronic health records contain rich information about drug history that would be valuable to the care of patients (eg, to prevent interaction with another medication and to track side effects), for epidemiology, or pharmaco-vigilance [4]. A major hurdle in the use of electronic health records is the format of the data. Up to $80 \%$ of relevant clinical information is present solely in the form of unstructured text, which represents a major barrier to the secondary use of this type of information $[5,6]$.

To overcome this issue, natural language processing techniques can be used to extract, normalize, and restructure drug-related information from clinical texts [6,7] and increase the information available for research and health care. Three approaches have been described for this task: expert knowledge modeling, machine learning, and hybrid methods (combining both).

The first approach relies on modeling expert knowledge using dictionaries or rules (ie, expert rules) such as MedEx, MedXN, or MedLEE based on lexicons or regular expressions [8-12]. Dictionary-based approaches allow for direct or approximate matching of terms from a dictionary or terminology. These approaches may offer poor results when the mentions used in texts deviate from the terms in the dictionary. Rule-based approaches allow for specific extractions but usually lack sensitivity and do not perform well on new data sets. Rule-based approaches also require domain experts to design and build the rules and are particularly time-consuming. In addition, expertise is rare and costly, which constitutes a severe bottleneck for the use of this type of method.

The second approach, using machine learning, has been developed in addition to expert approaches to extract medication name, dosage, frequency, duration, mode, reason for the intake and to detect adverse drug reactions $[13,14]$. Most systems included a conditional random field or a support vector machine for medication-related information extraction [15-18], 2 studies introduced bidirectional long short-term memory associated with conditional random field for named entity recognition and medication information extraction $[19,20]$, and another used a semisupervised model [21].

For the 2018 N2C2 shared task on medication extraction in electronic health records [22], several systems were proposed. The data set used in the challenge consisted of 505 discharge summaries extracted from the MIMIC-III database [23]. This data set contained 16,225 drug mentions in the training set and a total of 50,951 entity annotations again in the training set. Among the best-performing algorithms, bidirectional long short term memory and bidirectional long short term memory with conditional random field architectures were popular [24-27]. Some systems combined attention mechanisms [28] or convolutional neural networks [27]. Others combined classic entity extraction systems such as cTakes with classifiers such as support vector machines [29]. Ensemble approaches, combining multiple classifiers were also proposed [24-26,30].

At the conjunction of machine learning and expert rules, hybrid approaches can leverage the frugality of expert rules (in terms of data needs) and the flexibility and generalizability of machine learning. Examples include identifying medication heading using a conditional random field for named entity identification and a support vector machine to classify relations combined with a rule-based context engine [31]; a conditional random field and 2 bidirectional long short term memory-conditional random field models to extract handcrafted features [25]; and using expert rules and a knowledge base to enrich text, then using a bidirectional long short term memory with attention to perform the medication extraction in electronic health records [28]. These approaches were designed for text written in English. To the best of our knowledge, there are only a few studies [32,33] on French corpora: Deleger et al [32] used a rule-based system, and Lerner et al [33] developed a hybrid system that associated expert rules using terminology and bidirectional gated recurrent units with a conditional random field.

In recent years, the adoption of word embedding methods has led to a significant increase in the level of performance achievable by many natural language processing tasks [34]. Word embeddings use dense vector representation of the vocabulary. Interestingly, word embeddings are computed using large amounts of unannotated data (eg, Wikipedia). In static word embeddings, a token is represented by a static numeric vector. Recently, contextual word embedding methods have appeared, such as embedding for language model [35]. Contextual word embeddings provide a varying representation of the tokens with regard to the context in the text. Contextual word embeddings lead to richer representations and help to improve the performance in clinical concept extraction tasks [36]; results further improve when semantic information is incorporated [37].

In this work, we aimed to extract medication-related information from clinical narratives written in French in a real-world setting (ie, with documents directly extracted from a clinical data warehouse). Once extracted, such information can be restructured to be used for different purposes (eg, clinical epidemiology, monitoring, pharmaco-epidemiology, adverse drug reaction detection). Our purpose was two-fold: (1) We aimed to develop a gold standard data set of annotated clinical documents in French, along with an annotation guide, and (2) we aimed to develop a hybrid approach combining an association of knowledge base and expert rules, contextualized word embeddings training on clinical text, and a deep learning model based on bidirectional long short term memory-conditional random field. 


\section{Methods}

\section{Data}

\section{Source}

We leveraged the clinical data warehouse of the Assistance Publique-Hôpitaux de Paris (AP-HP), grouping data collected from 39 hospitals to build a data set of 1 million documents [38]. These clinical reports were medical prescriptions, discharge reports, examinations, observation reports, and emergency visits randomly selected from the clinical data warehouse.

\section{Annotated Data Set}

We created an annotated data set for training and evaluation. We iteratively developed an annotation guide during the first phase of annotation. A small portion of the extracted data set (320 documents) was manually annotated by 3 medical doctors using an annotation tool [39]. The annotations were converted to the inside, outside, beginning (IOB) standard. Tokens that refer to an entity were labeled B-entity_type for the first token and then I-entity_type, tokens outside entities mention are labeled $\mathrm{O}$. We split the 320 annotated clinical notes in a training set $(n=216)$, a development set $(n=24)$, and a test set $(n=80)$.

\section{Knowledge Base for Drug Names}

We relied upon 2 French databases_-Base de données publique des medicaments (a publicly accessible, National drug database)[40] and OpenMedic, a database from the national medical insurance agency [41]. These 2 databases contain all the drugs distributed in France during a given year. They were mapped to the Anatomical Therapeutic Chemical classification system. We extracted data from 2015 to 2019 and created a curated and unified dictionary of drug mentions.

The corpus can be made available on the condition that a research project is accepted by the scientific and ethics committee of the AP-HP health data warehouse.

After preprocessing, the text was preannotated using a set of expert handcrafted rules, then the texts were embedded using contextual word embeddings trained on a large corpus of clinical texts. The preannotations and the embedded texts were input into a bidirectional long short term memory-conditional random field to produce the final annotations (Figure 1; Figure 2).

Figure 1. General architecture of the model. BiLSTM: bidirectional long short term memory; CRF: conditional random field.

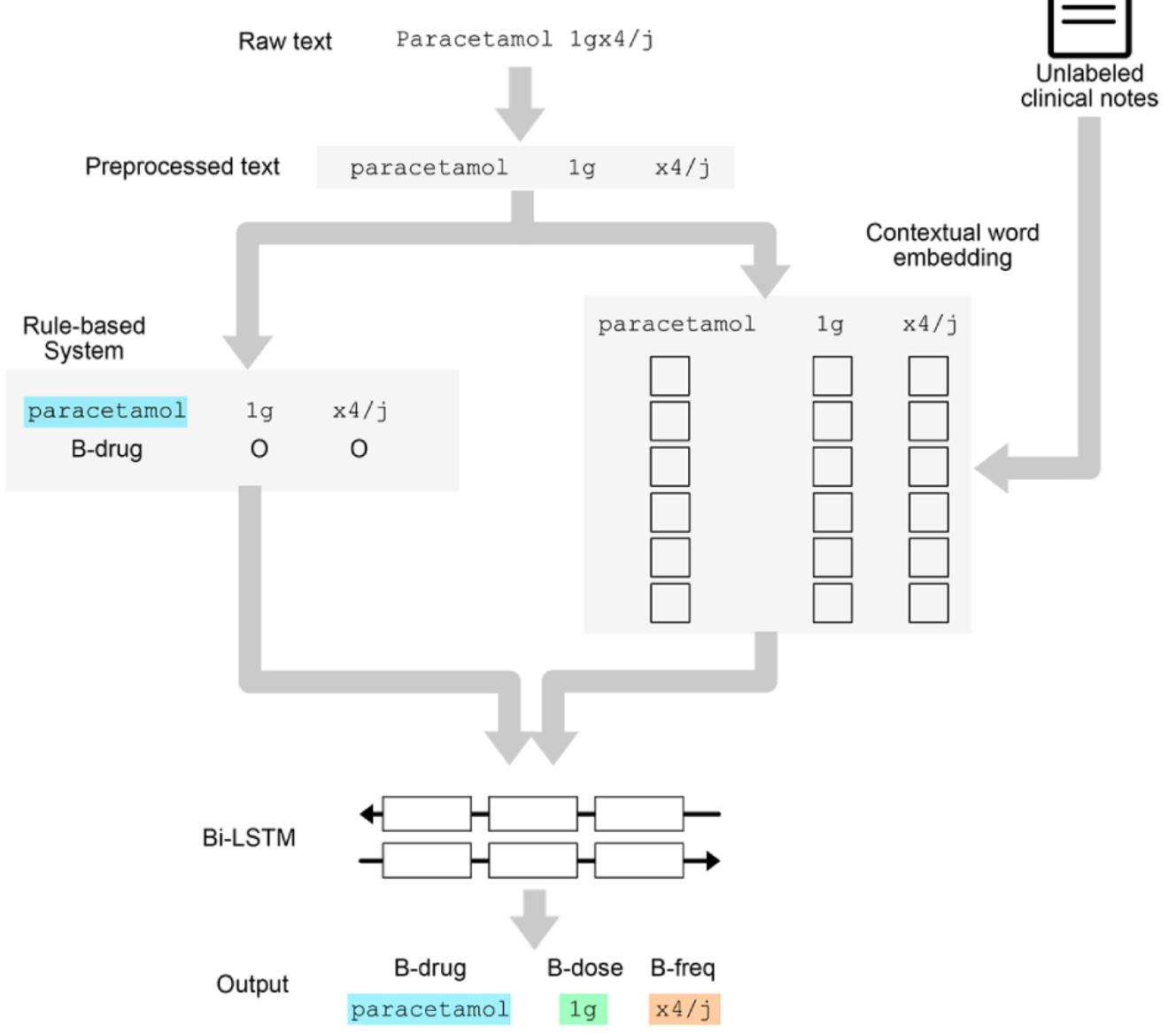


Figure 2. Annotation process with automatic annotation and completion with manual annotation.

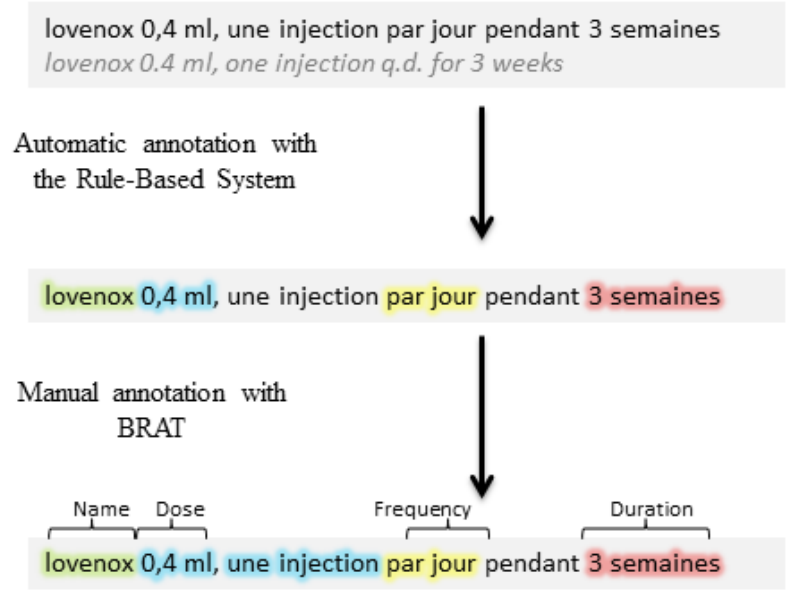

bolus de solumedrol iv debute en $16 / 04 / 1989$ puis $1 \mathrm{mg} / \mathrm{kg} / \mathrm{j}$ bolus IV of solumedrol started in 16/04/1989 and then $1 \mathrm{mg} / \mathrm{kg} /$ day

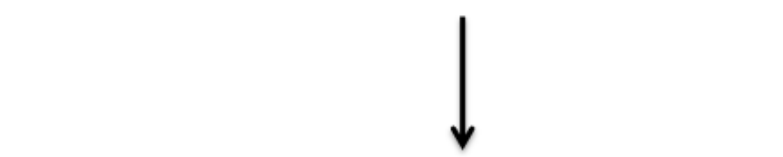

bolus de corticoides iv debute en $16 / 04 / 1989$ puis $1 \mathrm{mg} / \mathrm{kg} / \mathrm{j}$

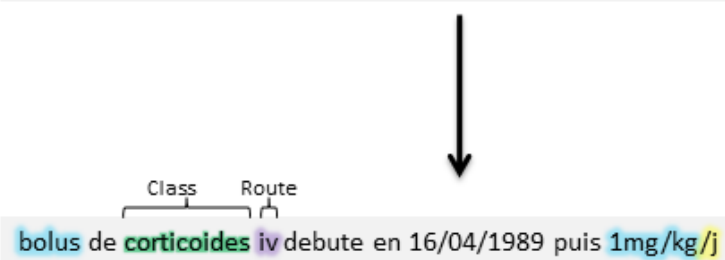

set of attributes related to the drug mentions: dosage, frequency, duration, route, and condition of administration. A detailed description of the types of entities is provided in Table 1.

\section{Task Definition}

We aimed to identify medication-related information in clinical documents in French. We were interested in drug names and a

Table 1. Description of the task.

\begin{tabular}{lll}
\hline Type & Description & Examples \\
\hline Medication name & $\begin{array}{l}\text { Descriptions that denote any medication, active molecule, associ- } \\
\text { ation or protocol }\end{array}$ & doliprane, paracetamol, augmentin \\
Medication class & $\begin{array}{l}\text { Descriptions that denote any Anatomical Therapeutic Chemical } \\
\text { class or common therapy }\end{array}$ & 3 mg, 2 tablets \\
Dosage & Dose or concentration of medication in prescription & 3 per day, every morning \\
Frequency & Frequency of medication administration & 3 weeks, until the surgery \\
Duration & Time range for the administration & intravenous, per os \\
Route & Medication administration mode & if pain, if infection \\
Condition & The event which provokes the administration &
\end{tabular}

\section{Preprocessing}

We preprocessed the input texts as described in Textbox 1.

Textbox 1. Text preprocessing.

Steps

- $\quad$ Removing acronym points and replacing decimal points by comma

- Removing break lines added during documents conversion to text

- Removing accents

- Replacing apostrophes by spaces

- Detecting sentence boundaries: remaining points or break lines without transitive verbs, preposition or coordinating conjunctions.

- Detecting word boundaries and tokenization: sequence of alphanumeric characters or a repetition of a unique nonalphanumeric characters

\section{Rule-Based Module}

The overall approach was organized as follows: we first identified a drug mention or a drug-class mention with the knowledge-based dictionary using exact matching. The choice of exact matching for this step was driven by maximizing the precision of the annotations in this preannotation step. Then, using the identified mention as an anchor, we extended the search to the attributes of this mention (ie, frequency, dosage, duration, mode of administration, and condition of administration) in the area surrounding the seed mention. The attributes were detected using a set of handcrafted rules using 
regular expressions. Examples of the rules are described in Table S1 of Multimedia Appendix 1. At this stage, the annotated entities were identified by their position and length relative to the beginning of the document. For the next steps, the annotations were converted to the IOB standard. The output of the rule-based system was used for preannotating the documents before the manual annotation step to speed up the annotation process of the gold standard data set and to serve as extra features to the input of the deep-learning module.

\section{Deep Learning Module}

\section{Overview}

We designed an approach leveraging deep neural networks. We tested 3 types of word embeddings-skip-gram [42], FastText embeddings [43], and embedding for language model [35]—and 2 neural network architectures-bidirectional long short term memory and bidirectional long short term memory-conditional random field.

\section{Embeddings}

We evaluated the impact of the word embeddings on the performance of the model. Our baseline was created using a skip-gram embedding trained on the training set only. We also considered FastText embedding (skip-gram model augmented with sub-word information) trained on a corpus of 1 million documents. Finally, we used embedding for language model embeddings, trained on 100,000 clinical notes that were contextualized embeddings computed through the internal states of a large bidirectional language model. The embeddings were kept fixed during model training.

\section{Combination of the Rule-Based System Output}

The output of the rule-based system was converted to the IOB standard. Then, this information was added as features to the input of the deep-learning module by concatenation with the word-embedding vectors.

\section{Models}

We used a deep recurrent neural network composed of long short-term memory units [44]. Specifically, we used bidirectional long short term memory composed of 2 concatenated long short term memory layers-one reading the input sequence forward, and another one reading the input sequence backward - allowing the model to take advantage of the context on the left and the right of a token when computing the latent states. The final prediction layer was either a standard dense layer with softmax or a conditional random field such as that in [19].

\section{Implementation and Optimization of Hyperparameters}

We implemented all the models using Keras and Keras-contrib [45] libraries using Python (version 3) with a TensorFlow backend [46]. We trained our models for 50 epochs, using an ADAM optimizer [47] with a learning rate of 0.001 and early stopping with a patience of 8 epochs. We applied a decrease of learning rate on plateau using a factor of 0.1 . For models with a final dense layer, we used categorical cross-entropy loss and softmax activation. For the models with conditional random field, we used marginal optimization and categorical cross-entropy loss. We tuned (using Hyperas version 0.4) the following hyperparameters using a random search with 15 iterations on the parameter space: batch size: 64,128 ; long short term memory size: $128,256,512$; dropout before and after long short term memory; and recurrent dropout: 0.0, 0.1, 0.2, 0.3, 0.5, 0.6, 0.7 (Table S2, Multimedia Appendix 1). All models were trained using NVIDIA P40 GPUs (3840 CUDA cores, 24 GB of DDRAM).

\section{Evaluation}

\section{Models}

We compared the performance of the rule-based system only, bidirectional long short term memory only, and rule-based system plus bidirectional long short term memory (with and without conditional random field). For bidirectional long short term memory with and without conditional random field models, we tested the impact of adding FastText embeddings or embedding for language model embeddings.

\section{Metrics}

We considered an extracted token to be a true positive if it was annotated with the correct category, a false positive if it was falsely annotated with respect to the evaluated class, and a false negative if it was not annotated or if it was annotated with an incorrect class. We computed the precision, recall, and F-measure to evaluate each model, microaveraging over all entries (Multimedia Appendix 2)

We also used the slot error rate metric. A slot corresponded to a mention of an entity (ie, a sequence of B and I tokens of the same class), a deletion was a missing slot, an addition was a slot that had been incorrectly added, a substitution or type error was a class that had been replaced by another class, and a frontier error was a token that had been added or removed at the end or the start of the slot [48].

\section{Results}

\section{Annotated Data Set}

The labeled data set contained 320 clinical notes and 19,957 sentences with 173,796 words. Training, development, and test sets included 216, 24, and 80 clinical notes with 13,737, 1373, and 4847 sentences, respectively. Table 2 summarizes the number of tokens and slots for each class in each data set. 
Table 2. Number of slots and tokens for each class per data set.

\begin{tabular}{|c|c|c|c|c|c|c|}
\hline \multirow[t]{2}{*}{ Label } & \multicolumn{2}{|l|}{ Train } & \multicolumn{2}{|c|}{ Development } & \multicolumn{2}{|l|}{ Test } \\
\hline & Tokens & Slots & Tokens & Slots & Tokens & Slots \\
\hline Medication name & 1385 & 1227 & 146 & 143 & 450 & 398 \\
\hline Medication class & 309 & 228 & 38 & 30 & 97 & 76 \\
\hline Dosage & 1366 & 761 & 115 & 62 & 606 & 311 \\
\hline Duration & 161 & 70 & 26 & 13 & 68 & 37 \\
\hline Route & 95 & 85 & 8 & 8 & 69 & 55 \\
\hline Condition & 192 & 61 & 9 & 3 & 89 & 28 \\
\hline
\end{tabular}

\section{Overall Comparison of the Models}

Table 3 summarizes the results of the different models. Overall, the best models were the hybrid models combining rule-based system, text embedding with embedding for language model, and bidirectional long short term memory (F-measure: 89.86). It had the lowest slot error rate $(0.19)$ with a minimal deletion rate $(0.05)$

The bidirectional long short term memory with baseline embedding had the worst results (F-measure: 73.93). Adding
FastText and embedding for language model trained on external data sets increased the F-measure by 14.15 and 9.81 points respectively. Combining rule-based system and bidirectional long short term memory increased the F-measure by 14.1 points.

The rule-based system alone had the highest precision (94.67) with the lowest insertion (0.03) and frontier (0.04) error rates. It had the second-lowest type error rate $(0.02)$ but one of the highest deletion error rates (0.23). Adding bidirectional long short term memory and embedding for language model to the rule-based system increased the F-measure by 10.45 points.

Table 3. Overall medication component information predictions metrics by models.

\begin{tabular}{|c|c|c|c|c|c|c|c|c|}
\hline Model $^{\mathrm{a}}$ & F-measure & Precision & Recall & $\begin{array}{l}\text { Slot error } \\
\text { rate }\end{array}$ & $\begin{array}{l}\text { Insertion } \\
\text { error rate }\end{array}$ & $\begin{array}{l}\text { Deletion } \\
\text { error rate }\end{array}$ & $\begin{array}{l}\text { Type error } \\
\text { rate }\end{array}$ & $\begin{array}{l}\text { Frontier } \\
\text { error rate }\end{array}$ \\
\hline $\mathrm{RBS}^{\mathrm{b}}$ & 79.41 & 94.67 & 72.28 & 0.29 & 0.03 & 0.23 & 0.02 & 0.04 \\
\hline BiLSTM $^{c}$ & 73.93 & 83.89 & 67.57 & 0.45 & 0.09 & 0.25 & 0.07 & 0.15 \\
\hline BiLSTM + FT & 88.08 & 89.48 & 87.17 & 0.21 & 0.07 & 0.08 & 0.03 & 0.09 \\
\hline $\mathrm{BiLSTM}+\mathrm{ELMo}^{\mathrm{e}}$ & 88.03 & 88.81 & 87.38 & 0.24 & 0.1 & 0.08 & 0.03 & 0.1 \\
\hline BiLSTM + RBS & 83.74 & 88.46 & 80.24 & 0.27 & 0.08 & 0.13 & 0.03 & 0.09 \\
\hline BiLSTM + FT + RBS & 88.18 & 91.73 & 85.54 & 0.21 & 0.07 & 0.09 & 0.01 & 0.07 \\
\hline BiLSTM + ELMo + RBS & 89.86 & 90.83 & 89.17 & 0.19 & 0.09 & 0.05 & 0.03 & 0.08 \\
\hline BiLSTM-CRF $^{\mathrm{f}}$ & 70.12 & 79.04 & 65.57 & 0.53 & 0.11 & 0.26 & 0.11 & 0.21 \\
\hline BiLSTM-CRF + FT & 87.16 & 88.58 & 86.41 & 0.25 & 0.09 & 0.08 & 0.03 & 0.12 \\
\hline BiLSTM-CRF + ELMo & 88.66 & 87.95 & 89.44 & 0.23 & 0.11 & 0.06 & 0.02 & 0.11 \\
\hline BiLSTM-CRF + RBS & 84.16 & 88.56 & 80.73 & 0.27 & 0.09 & 0.13 & 0.03 & 0.09 \\
\hline BiLSTM-CRF + FT + RBS & 87.74 & 89.72 & 86.25 & 0.22 & 0.08 & 0.08 & 0.02 & 0.09 \\
\hline BiLSTM-CRF + ELMo + RBS & 89.3 & 90.4 & 88.31 & 0.20 & 0.08 & 0.06 & 0.02 & 0.09 \\
\hline
\end{tabular}

${ }^{a}$ Models are described according to their components; if neither ELMo nor FT is mentioned, then we used skip-gram embedding.

${ }^{b}$ RBS: rule-based system (ie, the outputs are added as extra features to the input of the deep learning module).

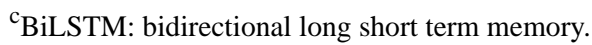

${ }^{\mathrm{d}}$ FT: FastText embedding.

${ }^{\mathrm{e}}$ ELMo: embedding for language model.

${ }^{\mathrm{f}} \mathrm{CRF}$ : conditional random field.

\section{Comparison by Type of Annotation}

Table 4 summarizes the metrics of the different models by type of entities. The rule-based system alone had the lowest
F-measure for every class due to a very low recall (medication class: 7.22), but it had the highest precision for all classes with the exception of medication name and duration. Associating the rule-based system to a bidirectional long short term memory 
increased medication name, medication class, dosage, and condition metrics (F-measures: 3.13, 3.12, 2.06, and 6.26, respectively) but decreased the F-measure for frequency, duration, and route (F-measures: $-1,-3.38$, and -2.66, respectively).

Table 4. Medication information predictions metrics results by models.

\begin{tabular}{llllllllll}
\hline Label & \multicolumn{1}{l}{ RBS } & \multicolumn{3}{c}{ BiLSTM + ELMo } & \multicolumn{3}{c}{ BiLSTM + ELMo + RBS } \\
& F-measure & Precision & Recall & F-measure & Precision & Recall & F-measure & Precision & Recall \\
\hline Medication name & 90.31 & 96.46 & 84.89 & 92.2 & 93.79 & 90.67 & 95.33 & 95.33 & 95.33 \\
Medication class & 13.33 & 87.5 & 7.22 & 62.3 & 66.28 & 58.76 & 64.36 & 61.9 & 67.01 \\
Dosage & 90.43 & 96.62 & 84.98 & 92.17 & 91.13 & 93.23 & 95.29 & 95.52 & 95.05 \\
Frequency & 86.13 & 98.89 & 76.28 & 92.8 & 93.3 & 92.31 & 92.24 & 93.04 & 91.45 \\
Duration & 48.89 & 49.25 & 48.53 & 82.17 & 86.89 & 77.94 & 78.79 & 81.25 & 76.47 \\
Route & 47.92 & 85.19 & 33.33 & 75.52 & 72.97 & 78.26 & 72.86 & 71.83 & 73.91 \\
Condition & 33.64 & 100 & 20.22 & 55.9 & 62.5 & 50.56 & 62.16 & 77.97 & 51.69 \\
\hline
\end{tabular}

${ }^{a}$ RBS: rule-based system

${ }^{\mathrm{b}}$ BiLSTM: bidirectional long short term memory.

${ }^{\mathrm{c}}$ ELMo: embedding for language models.

\section{Discussion}

\section{Principal Findings}

Our system achieved state-of-the-art performance for the task-an F-measure of 95.33 for medication names and an F-measure of 95.29 for dosage detection. Interestingly, these results were obtained using a data set representing only $10 \%$ of the size of similar data sets (N2C2 2018 shared task [22]). Combining expert knowledge (rule-based system) with a deep learning system increased the global F-measure, increased precision, increased recall, and decreased the slot error rate, having the most significant impacts on medication name, medication class, and dosage. While the rule-based system alone achieved the best precision and the worst recall, its association with the deep learning models helped to increase recall (for all information except condition) and increase precision (only for medication name, dosage, and condition of the intake). Adding a deep learning system with the embedding for language model on top of the rule-based system increased F-measures and recall for all categories. Adding a conditional random field layer increased the performance for the most frequent categories (ie, medication name, dosage, frequency). For other entities (ie, duration, route, condition), models with a conditional random field layer did not improve results (Multimedia Appendix 1). These results are consistent with those in the literature [18].

\section{Technical Significance}

It is interesting to note that leveraging the synergy between expert knowledge and deep learning allowed us to achieve performance comparable to state-of-the-art with only $10 \%$ of the data. Infusing knowledge into deep neural networks will probably be a key element in the future progress of the field. The use of externally trained embeddings is a first step in this direction given that they allow the incorporation of latent knowledge from large corpora into the models. The impact of contextualized embeddings proves that a more accurate representation is even more important. We can expect improved performance with more recent language representation approaches such as BERT [49] or XLNET [50]; however, the cost for fitting these types of models, in terms of computation, time, and data, will be a challenge for languages other than English, for which resources (ie, data) are less available. Therefore, it will be valuable to leverage other types of representations (such as ontologies) to infuse knowledge into neural networks. A possible path could be through specific embedding techniques such as Poincaré embeddings [51].

Our approach is highly versatile. It can be transposed to any language, as long as writing expert rules is feasible. We used regular expressions to this end, but any rule based can be used. Our approach is also transposable to other information extraction use cases (or even text classification).

\section{Clinical Significance}

The performance achieved by the system opens the way toward a large-scale use in real-life settings. We are currently developing an implementation to perform the medication information extraction at the scale of our institution. The versatility of the approach will enable its transposition to other types of clinical entities and information.

\section{Related Works}

Compared with systems developed on the I2B2 2009 medication data set, the performance of our system is competitive [31]. Regarding token metrics, we showed better performance (medication name, dosage, frequencies, and duration token-level F-measures: $+5.03,+4.49,+4.54,+28.89$, respectively). However, a direct comparison is difficult given that the data sets are different. First, we trained and evaluated our models on a different corpus of French clinical notes. Also, because of language differences, the annotation guidelines were not strictly identical.

In our corpus, the vast majority of medication name slots contained only one token $(48.7 \%$ of the medication names in the dictionary contain only one token), therefore, we can 
approximate a phrase-level F-measure using the token-level F-measure for medication names to compare with those in recent studies: Tao et al in 2018 reported a medication F-measure of 90.7 on the I2B 2 corpus, and we achieved an F-measure of 95.3 [21]. However, regarding the mode of administration, our result was lower (token-level F-measures: 72.9 vs 93.3).

In French-language clinical data sets, mode of administration mentions are less structured and more variable than those in English-language clinical text. Therefore, it is logical to see lower results in this field, and our findings were consistent with the findings from a previous study [32]. Moreover, we took the condition of the intake, and not the reason for the intake, into consideration (which is more specific), and we added a tag regarding the class name; therefore, overall F-measures cannot be compared. Compared with results from a study [33] using a different French-language corpus that obtained a token-level F-measure of 90.4, our system's raw results were higher. Comparisons should be made with caution because the corpus used in [33], though in the same language, was from a different source and contained only 147 documents.

The rule-based system offered the highest precision in most classes. The combination of deep learning and rule-based system could not maintain this high level of precision. One explanation could be that the performance of the rule-based system on the training set led the deep learning module to rely heavily on it. But when the rule-based system failed to generalize on the evaluation set, it caused a drop in accuracy in the hybrid system. This issue could be overcome by forcing the machine learning system to not exclusively rely on one source of information, contextual embedding or rule-based system features, by adding dropouts to the inputs.

Using a rule-based system associated with a deep learning model had two major benefits: the synergy between the rules and the machine learning increased the performance and the preannotation of the documents with the rules decreased the annotation time. Even if hybrid systems had already proved to be efficient [16,21,31,33,52], combining expert knowledge (rules) and latent knowledge (neural network), demonstrated a synergistic effect by increasing the performance in all metrics. It will be interesting to also evaluate approaches combining rules and deep learning in a reverse manner-first using a deep-learning model and refining the results using rules.

\section{Limitations and Perspectives}

We have several perspectives from which to continue this work. First, we did not reproduce our study on a standard corpus such as that of the I2B2 challenge. We would, therefore, have to redevelop all the expert rules for this English corpus. Second, the embedding for language model was trained on a set of 100,000 French clinical notes from a single hospital [53]. However, even with these limits, using the embedding for language model proved to be efficient. We can anticipate even better results with an embedding for language model trained on a larger and more diverse corpus. Finally, our study focused on recognizing medication information entities without extracting the relationships among them. Tao et al [21] described a way to model the relationships by predicting boundaries of utterances that contain related medication entities. We plan to extend this to all types of sentences in our corpus, independently of the number of medications mentions. To this end, we will build a multitask model to predict medication fields and relations. We will also predict medication event markers such as start, stop, increase, decrease, switch, or unique intake of medication. Moreover, we could also predict meta-attribute markers that would provide information on the experiencer (patient, family, other), temporality (in the past, present, or for the future), and certainty (eg, factual, suggested, hypothetical, conditional, negated, or contraindicated [54]).

\section{Conclusion}

The combination of expert rules, deep contextualized embedding (embedding for language model), and deep neural networks improved medication information extraction. This association achieved high performance on a heterogeneous corpus of French-language clinical reports, despite the data set's small size.

\section{Acknowledgments}

The authors thank the AP-HP health data warehouse for supporting this work.

\section{Conflicts of Interest}

None declared.

\section{Multimedia Appendix 1}

Supplementary tables. [DOCX File , 82 KB-Multimedia Appendix 1]

\section{Multimedia Appendix 2}

Formulas. [DOCX File, 79 KB-Multimedia Appendix 2]

\section{References}


1. Les dépenses de santé en 2017 - résultats des comptes de la santé - édition 2018. Direction de la recherche, des études, de l'évaluation et des statistiques. 2018. URL: https://drees.solidarites-sante.gouv.fr/publications/panoramas-de-la-drees/ les-depenses-de-sante-en-2017-resultats-des-comptes-de-la-sante [accessed 2019-10-01]

2. Olivier P, Boulbés O, Tubery M, Lauque D, Montastruc J, Lapeyre-Mestre M. Assessing the feasibility of using an adverse drug reaction preventability scale in clinical practice: a study in a French emergency department. Drug Saf 2002;25(14):1035-1044. [doi: 10.2165/00002018-200225140-00005] [Medline: 12408734]

3. Pirmohamed M, James S, Meakin S, Green C, Scott AK, Walley TJ, et al. Adverse drug reactions as cause of admission to hospital: prospective analysis of 18820 patients. BMJ 2004 Jul 03;329(7456):15-19 [FREE Full text] [doi: 10.1136/bmj.329.7456.15] [Medline: 15231615$]$

4. Zhou L, Mahoney LM, Shakurova A, Goss F, Chang FY, Bates DW, et al. How many medication orders are entered through free-text in EHRs?--a study on hypoglycemic agents. AMIA Annu Symp Proc 2012;2012:1079-1088 [FREE Full text] [Medline: 23304384]

5. Escudié JB, Jannot AS, Zapletal E, Cohen S, Malamut G, Burgun A, et al. Reviewing 741 patients records in two hours with FASTVISU. AMIA Annu Symp Proc 2015;2015:553-559 [FREE Full text] [Medline: 26958189]

6. Kreimeyer K, Foster M, Pandey A, Arya N, Halford G, Jones SF, et al. Natural language processing systems for capturing and standardizing unstructured clinical information: a systematic review. J Biomed Inform 2017 Sep;73:14-29 [FREE Full text] [doi: 10.1016/j.jbi.2017.07.012] [Medline: 28729030]

7. Gonzalez-Hernandez G, Sarker A, O'Connor K, Savova G. Capturing the patient's perspective: a review of advances in natural language processing of health-related text. Yearb Med Inform 2017 Aug;26(1):214-227 [FREE Full text] [doi: 10.15265/IY-2017-029] [Medline: 29063568]

8. Sirohi E, Peissig P. Study of effect of drug lexicons on medication extraction from electronic medical records. Pac Symp Biocomput 2005:308-318 [FREE Full text] [doi: 10.1142/9789812702456_0029] [Medline: 15759636]

9. Jagannathan V, Mullett CJ, Arbogast JG, Halbritter KA, Yellapragada D, Regulapati S, et al. Assessment of commercial NLP engines for medication information extraction from dictated clinical notes. Int J Med Inform 2009 Apr;78(4):284-291. [doi: 10.1016/j.ijmedinf.2008.08.006] [Medline: 18838293]

10. Hyun S, Johnson SB, Bakken S. Exploring the ability of natural language processing to extract data from nursing narratives. Comput Inform Nurs 2009;27(4):215-23; quiz 224 [FREE Full text] [doi: 10.1097/NCN.0b013e3181a91b58] [Medline: 19574746]

11. Xu H, Stenner SP, Doan S, Johnson KB, Waitman LR, Denny JC. MedEx: a medication information extraction system for clinical narratives. J Am Med Inform Assoc 2010 Jan 01;17(1):19-24. [doi: 10.1197/jamia.m3378]

12. Sohn S, Clark C, Halgrim SR, Murphy SP, Chute CG, Liu H. MedXN: an open source medication extraction and normalization tool for clinical text. J Am Med Inform Assoc 2014;21(5):858-865 [FREE Full text] [doi: 10.1136/amiajnl-2013-002190] [Medline: 24637954]

13. Wang Y, Wang L, Rastegar-Mojarad M, Moon S, Shen F, Afzal N, et al. Clinical information extraction applications: a literature review. J Biomed Inform 2018 Jan;77:34-49 [FREE Full text] [doi: 10.1016/j.jbi.2017.11.011] [Medline: 29162496]

14. Jagannatha A, Liu F, Liu W, Yu H. Overview of the first natural language processing challenge for extracting medication, indication, and adverse drug events from electronic health record notes (MADE 1.0). Drug Saf 2019 Jan;42(1):99-111 [FREE Full text] [doi: 10.1007/s40264-018-0762-z] [Medline: 30649735]

15. Doan S, Collier N, Xu H, Pham HD, Tu MP. Recognition of medication information from discharge summaries using ensembles of classifiers. BMC Med Inform Decis Mak 2012 May 07;12:36 [FREE Full text] [doi: 10.1186/1472-6947-12-36] [Medline: 22564405]

16. Li Q, Spooner SA, Kaiser M, Lingren N, Robbins J, Lingren T, et al. An end-to-end hybrid algorithm for automated medication discrepancy detection. BMC Med Inform Decis Mak 2015 May 06;15:37 [FREE Full text] [doi: 10.1186/s12911-015-0160-8] [Medline: 25943550]

17. Zhang Y, Xu J, Chen H, Wang J, Wu Y, Prakasam M, et al. Chemical named entity recognition in patents by domain knowledge and unsupervised feature learning. Database (Oxford) 2016;2016 [FREE Full text] [doi: 10.1093/database/baw049] [Medline: 27087307]

18. Lafferty J, McCallum A, Pereira F. Conditional random fields: probabilistic models for segmenting and labeling sequence data. In: Proceedings of the Eighteenth International Conference on Machine Learning.: Morgan Kaufmann Publishers Inc; 2001 Presented at: Eighteenth International Conference on Machine Learning; June 28-July 1; Williamstown, Massachusetts, USA p. 282-289. [doi: 10.5555/645530.655813]

19. Lample G, Ballesteros M, Subramanian S, Kawakami K, Dyer C. Neural architectures for named entity recognition. 2016 Presented at: Conference of the North American Chapter of the Association for Computational Linguistics: Human Language Technologies; June 12-17; San Diego, California p. 260-270. [doi: 10.18653/v1/N16-1030]

20. Sadikin M, Fanany MI, Basaruddin T. A new data representation based on training data characteristics to extract drug name entity in medical text. Comput Intell Neurosci 2016;2016:3483528. [doi: 10.1155/2016/3483528] [Medline: 27843447]

21. Tao C, Filannino M, Uzuner Ö. FABLE: a semi-supervised prescription information extraction system. AMIA Annu Symp Proc 2018;2018:1534-1543 [FREE Full text] [Medline: 30815199] 
22. Henry S, Buchan K, Filannino M, Stubbs A, Uzuner O. 2018 n2c2 shared task on adverse drug events and medication extraction in electronic health records. J Am Med Inform Assoc 2020 Jan 01;27(1):3-12 [FREE Full text] [doi: 10.1093/jamia/ocz166] [Medline: $\underline{31584655}$ ]

23. Johnson AEW, Pollard TJ, Shen L, Lehman LH, Feng M, Ghassemi M, et al. MIMIC-III, a freely accessible critical care database. Sci Data 2016 May 24;3:160035 [FREE Full text] [doi: 10.1038/sdata.2016.35] [Medline: 27219127]

24. Christopoulou F, Tran TT, Sahu SK, Miwa M, Ananiadou S. Adverse drug events and medication relation extraction in electronic health records with ensemble deep learning methods. J Am Med Inform Assoc 2020 Jan 01;27(1):39-46 [FREE Full text] [doi: 10.1093/jamia/ocz101] [Medline: $\underline{\text { 31390003] }}$

25. Dai H, Su C, Wu C. Adverse drug event and medication extraction in electronic health records via a cascading architecture with different sequence labeling models and word embeddings. J Am Med Inform Assoc 2020 Jan 01;27(1):47-55 [FREE Full text] [doi: 10.1093/jamia/ocz120] [Medline: $\underline{31334805]}$

26. Kim Y, Meystre SM. Ensemble method-based extraction of medication and related information from clinical texts. J Am Med Inform Assoc 2020 Jan 01;27(1):31-38 [FREE Full text] [doi: 10.1093/jamia/ocz100] [Medline: 31282932]

27. Yang X, Bian J, Fang R, Bjarnadottir R, Hogan W, Wu Y. Identifying relations of medications with adverse drug events using recurrent convolutional neural networks and gradient boosting. J Am Med Inform Assoc 2020 Jan 01;27(1):65-72 [FREE Full text] [doi: 10.1093/jamia/ocz144] [Medline: 31504605]

28. Chen L, Gu Y, Ji X, Sun Z, Li H, Gao Y, et al. Extracting medications and associated adverse drug events using a natural language processing system combining knowledge base and deep learning. J Am Med Inform Assoc 2020 Jan 01;27(1):56-64 [FREE Full text] [doi: 10.1093/jamia/ocz141] [Medline: 31591641$]$

29. Miller T, Geva A, Dligach D. Extracting adverse drug event information with minimal engineering. 2019 Presented at: 2nd Clinical Natural Language Processing Workshop; June 6-7; Minneapolis, Minnesota, USA p. 22-27. [doi:

10.18653/v1/W19-1903]

30. Xu J, Lee HJ, Ji Z, Wang J, Wei Q, Xu H. UTH_CCB system for adverse drug reaction extraction from drug labels track. 2017 Presented at: 2017 Text Analysis Conference; November 13-14; Gaithersburg, Maryland, USA.

31. Patrick J, Li M. High accuracy information extraction of medication information from clinical notes: 2009 i2b2 medication extraction challenge. J Am Med Inform Assoc 2010;17(5):524-527 [FREE Full text] [doi: 10.1136/jamia.2010.003939] [Medline: 20819856]

32. Deléger L, Grouin C, Zweigenbaum P. Extracting medication information from French clinical texts. Stud Health Technol Inform 2010;160(Pt 2):949-953. [Medline: 20841824]

33. Lerner I, Paris N, Tannier X. Terminologies augmented recurrent neural network model for clinical named entity recognition. J Biomed Inform 2020 Feb;102:103356 [FREE Full text] [doi: 10.1016/j.jbi.2019.103356] [Medline: 31837473]

34. Mikolov T, Chen K, Corrado G, Dean J. Efficient estimation of word representations in vector space. 2013 Presented at: 1st International Conference on Learning Representations; May 2-4; Scottsdale, Arizona, USA.

35. Peters M, Neumann M, Iyyer M, Gardner M, Clark C, Lee K. Deep contextualized word representations. In: Proceedings of the 2018 Conference of the North American Chapter of the Association for Computational Linguistics: Human Language Technologies, Volume 1 (Long Papers). 2018 Presented at: 2018 Conference of the North American Chapter of the Association for Computational Linguistics: Human Language Technologies; June 1-6; New Orleans, Louisiana, USA. [doi: $10.18653 / \mathrm{v} 1 / \mathrm{n} 18-1202]$

36. Zhu H, Paschalidis I, Tahmasebi A. Clinical concept extraction with contextual word embedding. arXiv. Preprint posted online on October 24, 2018 [FREE Full text]

37. Jiang M, Sanger T, Liu X. Combining contextualized embeddings and prior knowledge for clinical named entity recognition: evaluation study. JMIR Med Inform 2019 Nov 13;7(4):e14850 [FREE Full text] [doi: 10.2196/14850] [Medline: 31719024$]$

38. Daniel C, Serre P, Orlova N, Bréant S, Paris N, Griffon N. Initializing a hospital-wide data quality program. the AP-HP experience. Comput Methods Programs Biomed 2019 Nov;181:104804. [doi: 10.1016/j.cmpb.2018.10.016] [Medline: 30497872]

39. Stenetorp P, Pyysalo S, Topic G, Ohta T, Ananiadou S, Tsujii J. BRAT: a web-based tool for NLP-assisted text annotation. 2012 Presented at: 13th Conference of the European Chapter of the Association for Computational Linguistics; April 23-27; Avignon, France p. 102-107 URL: http://dl.acm.org/citation.cfm?id=2380921.2380942

40. Base de données publique des médicaments. Ministère des Affaires Sociales et de la Santé. URL: http://base-donnees-publique. medicaments.gouv.fr [accessed 2019-10-01]

41. Médicaments remboursés par l'Assurance Maladie. Plateforme Ouverte des Données Publiques Françaises. URL: https:/ /www.data.gouv.fr/fr/datasets/medicaments-rembourses-par-lassurance-maladie/ [accessed 2019-10-01]

42. Mikolov T, Sutskever I, Chen K, Corrado G, Dean J. Distributed representations of words and phrases and their compositionality. In: Proceedings of the 26th International Conference on Neural Information Processing Systems - Volume 2. 2013 Presented at: 26th International Conference on Neural Information Processing System; 2013; Lake Tahoe, Nevada, USA p. 3111-3119 URL: http://papers.nips.cc/paper/ 5021-distributed-representations-of-words-and-phrases-and-their-compositionality.pdf [doi: 10.5555/2999792.2999959]

43. Bojanowski P, Grave E, Joulin A, Mikolov T. Enriching word vectors with subword information. Trans Assoc Comput Linguist 2017 Dec;5:135-146. [doi: 10.1162/tacl a 00051] 
44. Hochreiter S, Schmidhuber J. Long short-term memory. Neural Comput 1997 Nov 15;9(8):1735-1780. [doi: 10.1162/neco.1997.9.8.1735] [Medline: 9377276]

45. Chollet F. Keras: the python deep learning library. Astrophysics Source Code Library. 2018. URL: https://ui. adsabs.harvard.edu/abs/2018ascl.soft06022C [accessed 2019-10-01]

46. Abadi M, Barham P, Chen J, Chen Z, Davis A, Dean J, et al. TensorFlow: a system for large-scale machine learning. In: Proceedings of the 12th USENIX Symposium on Operating Systems Design and Implementation. 2016 Presented at: 12 th USENIX Symposium on Operating Systems Design and Implementation; November 2-4; Savannah, Georgia, USA.

47. Kingma D, Ba J. Adam: a method for stochastic optimization. arXiv. Preprint posted online on January 30, 2017 [FREE Full text]

48. Makhoul J, Kubala F, Schwartz R, Weischedel R. Performance measures for information extraction. In: Proceedings of DARPA Broadcast News Workshop. 1999 Presented at: DARPA Broadcast News Workshop; June 29; Herndon, Virginia, USA p. 249-252.

49. Devlin J, Chang M, Lee K, Toutanova K. BERT: pretraining of deep bidirectional transformers for language understanding. arXiv. Preprint posted online on May 24, 2019 [FREE Full text]

50. Yang Z, Dai Z, Yang Y, Carbonell J, Salakhutdinov R, Le Q. XLNet: generalized autoregressive pretraining for language understanding. 2019 Presented at: Advances in Neural Information Processing Systems 32; December 8-14; Vancouver, British Columbia, Canada.

51. Agarwal K, Eftimov T, Addanki R, Choudhury S, Tamang S, Rallo R. Snomed2Vec: random walk and Poincaré embeddings of a clinical knowledge base for health care analytics. arXiv. Preprint posted online on July 19, 2019 [FREE Full text]

52. Uzuner O, Solti I, Cadag E. Extracting medication information from clinical text. J Am Med Inform Assoc 2010;17(5):514-518 [FREE Full text] [doi: 10.1136/jamia.2010.003947] [Medline: 20819854]

53. Neuraz A, Llanos L, Burgun A, Rosset S. Natural language understanding for task oriented dialog in the biomedical domain in a low resources context. arXiv. Preprint posted online on November 29, 2018 [FREE Full text]

54. Jouffroy J, Feldman S, Neuraz A. Medication extraction annotation guide for french clinical texts. Équipe 22 GitHub. 2019 Jun 06. URL: https://equipe22.github.io/medExtAnnotation/ [accessed 2019-10-01]

\title{
Abbreviations \\ AP-HP: Assistance Publique-Hôpitaux de Paris \\ IOB: inside, outside, beginning
}

\author{
Edited by C Lovis; submitted 23.01.20; peer-reviewed by S Cossin, L Wang, S Kim; comments to author 06.09.20; revised version \\ received 29.12.20; accepted 20.01.21; published 16.03.21 \\ Please cite as: \\ Jouffroy J, Feldman SF, Lerner I, Rance B, Burgun A, Neuraz A \\ Hybrid Deep Learning for Medication-Related Information Extraction From Clinical Texts in French: MedExt Algorithm Development \\ Study \\ JMIR Med Inform 2021;9(3):e17934 \\ URL: https://medinform.jmir.org/2021/3/e17934 \\ doi: $\underline{10.2196 / 17934}$ \\ PMID: 33724196
}

CJordan Jouffroy, Sarah F Feldman, Ivan Lerner, Bastien Rance, Anita Burgun, Antoine Neuraz. Originally published in JMIR Medical Informatics (http://medinform.jmir.org), 16.03.2021. This is an open-access article distributed under the terms of the Creative Commons Attribution License (https://creativecommons.org/licenses/by/4.0/), which permits unrestricted use, distribution, and reproduction in any medium, provided the original work, first published in JMIR Medical Informatics, is properly cited. The complete bibliographic information, a link to the original publication on http://medinform.jmir.org/, as well as this copyright and license information must be included. 